\title{
Neural Mapping of Direction and Frequency in the Cricket Cercal Sensory System
}

\author{
Sussan Paydar, ${ }^{2}$ Caitlin A. Doan, ${ }^{2}$ and Gwen A. Jacobs ${ }^{1}$ \\ ${ }^{1}$ Center for Computational Biology, Montana State University, Bozeman, Montana 59717, and 2Department of Molecular \\ and Cell Biology, University of California, Berkeley, California 94720
}

Primary mechanosensory receptors and interneurons in the cricket cercal sensory system are sensitive to the direction and frequency of air current stimuli. Receptors innervating long mechanoreceptor hairs $(>1000 \mu \mathrm{m})$ are most sensitive to lowfrequency air currents $(<150 \mathrm{~Hz})$; receptors innervating medium-length hairs $(900-500 \mu \mathrm{m})$ are most sensitive to higher frequency ranges $(150-400 \mathrm{~Hz}$ ). Previous studies demonstrated that the projection pattern of the synaptic arborizations of long hair receptor afferents form a continuous map of air current direction within the terminal abdominal ganglion (Jacobs and Theunissen, 1996). We demonstrate here that the projection pattern of the medium-length hair afferents also forms a continuous map of stimulus direction. However, the afferents from the long and medium-length hair afferents show very little spatial segregation with respect to their frequency sensitivity. The possible functional significance of this small degree of spatial segregation was investigated, by calculating the relative overlap between the long and medium-length hair afferents with the dendrites of two interneurons that are known to have different frequency sensitivities. Both interneurons were shown to have nearly equal anatomical overlap with long and medium hair afferents. Thus, the differential overlap of these interneurons with the two different classes of afferents was not adequate to explain the observed frequency selectivity of the interneurons. Other mechanisms such as selective connectivity between subsets of afferents and interneurons and/or differences in interneuron biophysical properties must play a role in establishing the frequency selectivities of these interneurons.

Key words: sensory maps; sensory system; insect; functional neuroanatomy; three-dimensional reconstruction; mechanosensory receptors
A basic organizational principle of most sensory systems is the representation of stimulus parameters within neural maps. Neural maps are ensembles of neurons in which specific parameters of the sensory stimulus are represented in a continuous manner across the neural ensemble. Many neural maps represent multiple stimulus parameters. In the visual cortex, for example, maps of orientation selectivity, ocular dominance, and direction selectivity coexist in the same regions of cortex. How do such complex neural maps emerge from the anatomical projection pattern of an ensemble of neurons? How do the anatomical features of such maps constrain and/or simplify the extraction of information by the neurons forming the next processing stage?

We addressed these questions in the cercal sensory system of the cricket. In this system, primary sensory neurons innervate mechanoreceptor hairs on two abdominal sensory appendages, called cerci. The sensory neurons are activated by air current stimuli and display differential sensitivity to variation in direction and frequency parameters (Kamper and Kleindienst, 1990; Warland et al., 1991; Landolfa and Jacobs, 1995; Landolfa and Miller, 1995; Roddey and Jacobs, 1996). The axons of the primary sensory neurons project in an orderly array into the terminal abdominal ganglion, forming a continuous representation of air

Received July 27, 1998; revised Dec. 7, 1998; accepted Dec. 9, 1998.

This work was supported by National Science Foundation Grant IBN-9796117 and National Institutes of Health Grant R29 NS29847 to G.A.J. We thank Sandy Pittendrigh and Kelli Hodge for expert help with the graphics and John Miller for critically reading this manuscript.

Correspondence should be addressed to Dr. Gwen A. Jacobs, Center for Computational Biology, 30 AJM Johnson Hall, Montana State University, Bozeman, MT 59717.

Copyright (C) 1999 Society for Neuroscience $\quad 0270-6474 / 99 / 191771-11 \$ 05.00 / 0$ current direction (Bacon and Murphey, 1984; Troyer et al., 1994; Jacobs and Theunissen, 1996). Neurons with similar directional tuning overlap anatomically. The spatial segregation between arbors increases within the map as the difference in directional tuning increases. The sensory neurons make excitatory synaptic connections onto interneurons that project to higher levels of the nervous system. These interneurons encode information about the direction and frequency of air currents (Kanou and Shimozawa, 1984; Jacobs et al., 1986; Miller et al., 1991; Theunissen et al., 1996).

Although the afferents of the sensory receptors are known to form a neural map of stimulus direction, it is not known how stimulus frequency is represented within the terminal ganglion. Are afferent arborizations segregated with respect to their frequency selectivities? Does a continuous map of stimulus frequency coexist with the map of stimulus direction within the ganglion? To address these questions, the anatomical projection patterns of two populations of sensory neurons were analyzed quantitatively. Both populations of neurons project to the same general region of the terminal ganglion, and both populations are sensitive to the same range of air current directions. However, the two afferent populations are sensitive to different ranges of air current frequency. One population is most sensitive to air currents having low frequencies $(0-150 \mathrm{~Hz})$, whereas the other is tuned to higher frequencies $(150-400 \mathrm{~Hz}$ ) (Roddey and Jacobs, 1996). In this paper, we determined how multiple stimulus parameters are represented anatomically within the ganglion by comparing the projection patterns of these two populations of sensory neurons. We also tested the hypothesis that the frequency-tuning properties of interneurons, which receive input 
from these sensory neurons, depend on specific anatomical relationships between the interneurons and the two populations of sensory neurons.

\section{MATERIALS AND METHODS}

The location of each mechanoreceptor as well as its functional characteristics are relatively invariant among animals (Landolfa and Jacobs, 1995). Thus it is possible to identify hairs and measure their functional characteristics by direct observation through the dissecting microscope. The length of the mechanoreceptor, which determines its dynamic sensitivity, was measured with a calibrated ocular micrometer. Its directional sensitivity was determined by measuring the angle of the hair's movement axis with respect to the cercal axis and translating it to the body coordinate system (Landolfa and Jacobs, 1995). For each identified mechanoreceptor, the length and directional tuning was measured for 10 examples of that identified hair. The angular measurements across animals had an SD of $<2.5 \mu \mathrm{m}$.

Histological staining techniques. Adult female crickets were used within $24 \mathrm{hr}$ of their imaginal molt (Bassetts Cricket Ranch, Visalia, CA). Each individual afferent was stained with cobalt chloride and silver-intensified using methods developed by Bacon and Altman (1977), further modified by Johnson and Murphey (1985) and Jacobs and Nevin (1991). The interneurons were stained with cobalt chloride and developed using the same methods.

Computer reconstruction of stained neurons. The microscopy and computer techniques used to digitize stained cells were developed in our laboratory and have been described in detail elsewhere (Jacobs and Nevin, 1991). Briefly, a computer-controlled, digitizing light microscope is used as the data entry device. Tissue containing a single dye-filled nerve cell is mounted on the microscope stage, and the operator controls the precise movement of the neuron in three dimensions by means of three precision stepper motors, each mounted on a different axis of the mechanical microscope stage. The neuron is moved under the microscope so that its branches are traced under a video cursor, superimposed over a frame-grabbed video image of the tissue. Movements of the microscope stage are monitored by linear encoders mounted to the stage, each encoder having a resolution of $0.1 \mu \mathrm{m}$. A set of $x, y$, and $z$ coordinates are recorded for the end points of each dendritic segment, along with the mean diameter of the segment between the end points.

To compare neurons from different animals, three to five samples of each of the 13 representative $M$ afferents and one example of each interneuron [interneuron 10-3 (IN10-3) and IN9-3] were digitized. All digitized neurons were scaled and aligned to a common coordinate system using a suite of software programs called NeuroSys, developed in our laboratory. NeuroSys contains a relational database and a suite of morphological analysis and computational tools (http://www.nervana.montana.edu/NeuroSys). The information currently in the database consists of $>200$ receptors associated with two functional classes of afferents: those innervating the longest hairs on the cercus $(>500 \mu \mathrm{m}$ in length) and those innervating medium-length hairs (900-500 $\mu \mathrm{m}$ in length). We have constructed a three-dimensional model of this system, in the form of a probabilistic atlas, by combining three key morphological and functional characteristics of the receptor neurons: (1) their optimal stimulus directions, (2) their frequency-tuning characteristics, and (3) the spatial distribution of each afferent's synaptic sites within the cercal glomerulus. The database also contains reconstructions of a subset of the identified primary interneurons.

Estimating the total surface area of an afferent's synaptic field. Jacobs and Theunissen (1996) developed a method for quantifying the spatial distribution of the surface area of the terminal varicosities of primary sensory afferents. The same method was used here. Briefly, for each identified afferent type, three to five examples were taken from different animals. The terminal varicosities of each cell were transformed into a continuous function representing the anatomical location and density distribution of the varicosities of a given identified afferent. These density functions were visualized as three-dimensional space-filling clouds of points and used to calculate the location and spatial extent of the arborization of a single afferent. Details of statistical methods used for this procedure are described by Jacobs and Theunissen (1996).

Overlap measurements. The anatomical overlap between pairs of afferents or between interneurons and afferents was calculated as the intersection between the density functions of the two cells. For the interneurons, the density distribution of their terminal dendritic branches $(<2$ $\mu \mathrm{m}$ in diameter) was calculated in the same way as the afferent varicosities (for details, see Jacobs and Theunissen, 1996).

\section{RESULTS}

To clarify the presentation of the results, the following is a short description of the functional tuning properties of the primary sensory neurons described in this paper.

\section{Tuning properties of primary sensory neurons in the cercal sensory system}

Each wind-sensitive sensory neuron in the cercal system innervates a single filiform mechanoreceptive hair. The biomechanical properties of the mechanoreceptor determine how it moves in response to air currents and, in turn, determines the firing pattern of the sensory neuron. The mechanoreceptor hair is lodged in a socket that constrains its motion to a single plane. The directional tuning properties of the sensory neuron are thereby determined by the orientation of this plane of motion with respect to the cercus. Sensory neurons are also broadly tuned to air current frequency. The length of the mechanoreceptor determines the frequency-tuning properties of the sensory neurons (Kamper and Kleindienst, 1990; Landolfa and Jacobs, 1995; Landolfa and Miller, 1995). The mechanoreceptor hairs vary in length from $\sim 50 \mu \mathrm{m}$ to $1.5 \mathrm{~mm}$.

In this study we compared the anatomical projection patterns of two populations of afferents: those innervating the longest hairs on the cercus (the "L afferents," associated with mechanoreceptor hairs $>1000 \mu \mathrm{m}$ in length) and those innervating mediumlength hairs (the "M afferents," associated with hairs 700-950 $\mu \mathrm{m}$ in length). Several aspects of the anatomical projection patterns were analyzed quantitatively: arbor size, arbor position in the terminal abdominal ganglion, and amount of anatomical overlap with neighboring arbors. The goal of these experiments was to determine how stimulus direction and frequency parameters are represented anatomically within a neural map by testing whether the terminal arborizations of the $\mathrm{L}$ and $\mathrm{M}$ afferents are systematically and continuously segregated within the ganglion.

\section{Ensemble projection pattern of the medium hair afferents}

The axonal arborizations of 13 uniquely identified $M$ afferents were stained, digitally reconstructed, scaled, and aligned to our database of identified neurons. Figure 1 shows the axonal arborization of each identified $\mathrm{M}$ afferent within the terminal ganglion. All $\mathrm{M}$ afferents project into the terminal abdominal ganglion and arborize in specific locations according to their directional tuning characteristics. Both the location on the cercus and the directional sensitivities of these $\mathrm{M}$ afferents correspond to identified $\mathrm{L}$ afferents with the same locations and directional sensitivities described in previous work (Jacobs and Theunissen, 1996), with one exception. The exception is that one additional identified $\mathrm{M}$ afferent (Fig. 1, \#13) was reconstructed in the current study, whereas only 12 afferents were reconstructed in the initial study of the L afferents. Each corresponding pair of identified $\mathrm{L}$ and $\mathrm{M}$ afferents had the same directional tuning.

The location and branching structure of the axonal arbors of each identified $\mathrm{M}$ afferent was compared with identified $\mathrm{L}$ afferents with the same directionality. Figure 2 shows the branching patterns of three examples of $\mathrm{M}$ afferents (blue) and three $\mathrm{L}$ afferents of the same directional tuning (red). Thus the projection pattern of the population $M$ afferents is very similar to the projection pattern of $\mathrm{L}$ afferents. Each pair of corresponding afferents projects to the same general area, according to their common directional tuning characteristics.

However, there were small but significant differences in the 

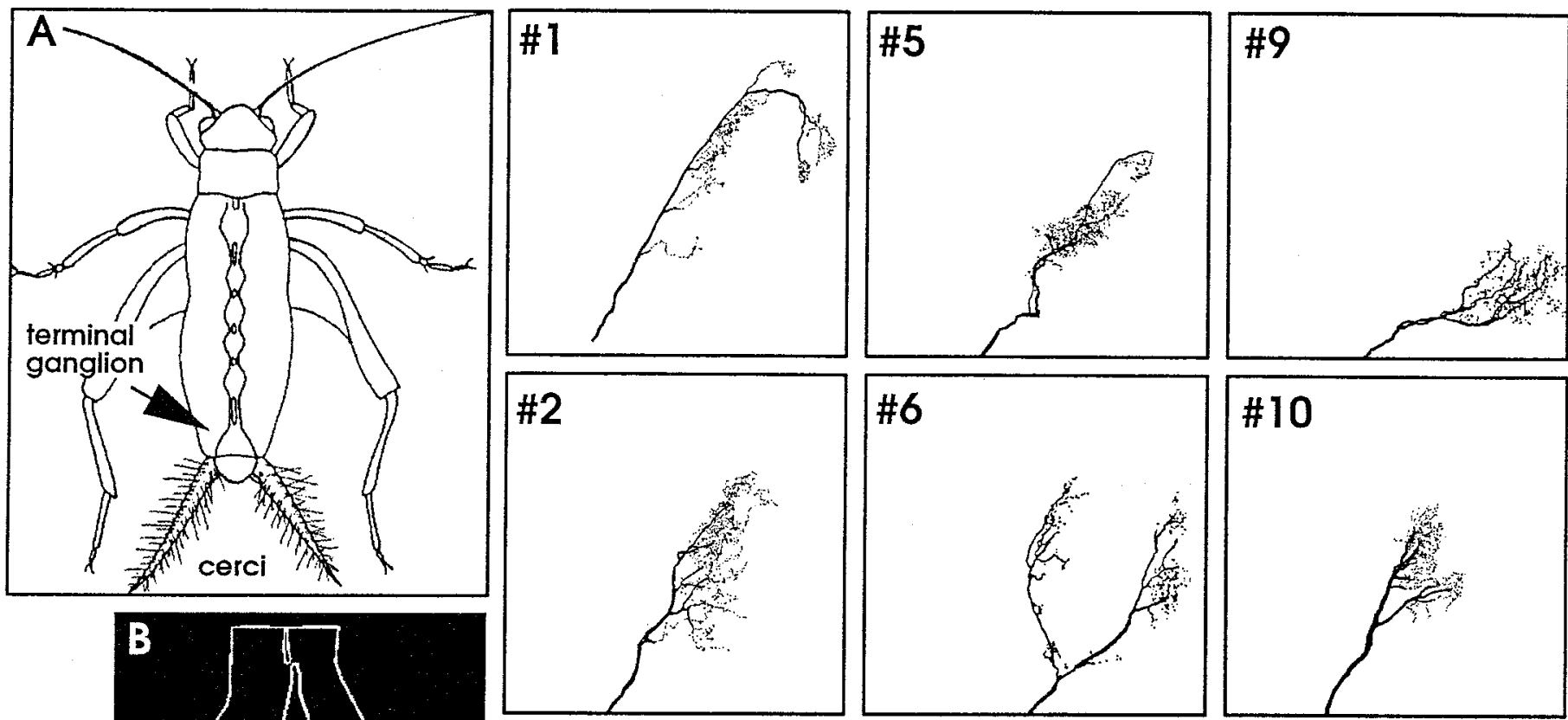

$\# 10$
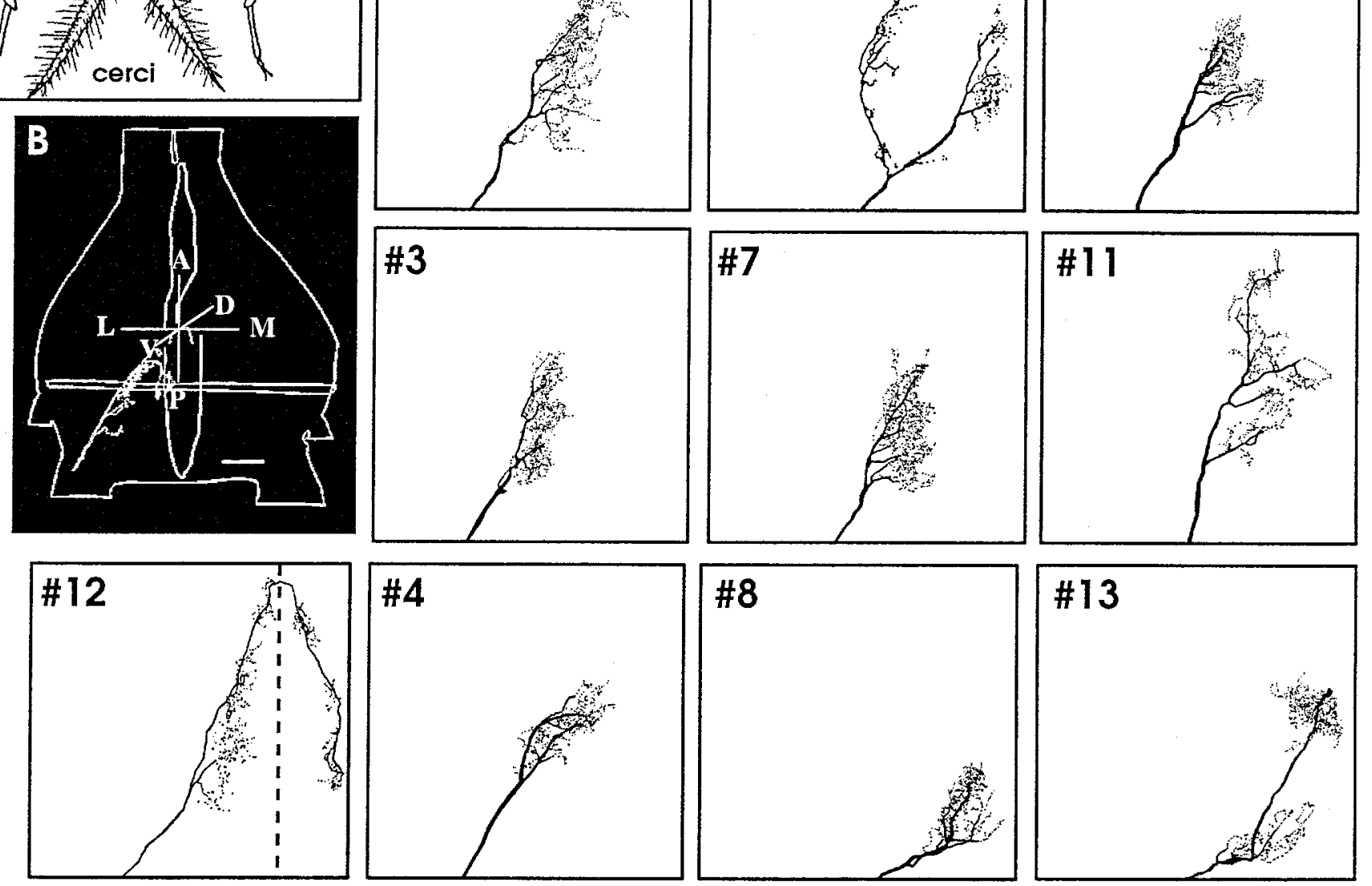

Figure 1. Terminal arborizations of 13 types of identified cercal sensory afferents within the terminal ganglion. $A$, Diagram of a cricket showing the location of the terminal abdominal ganglion and cerci. $B$, Computer reconstruction of the outline of the terminal ganglion showing afferent type \# 1 as an example, dorsal view. The vertical and horizontal loops inside the ganglion are fiducial marks and are used for scaling and aligning. Afferent type \#12 (also referred as $\mathrm{X}$ ) is the only afferent that projects bilaterally. All other panels show the remaining 12 classes of afferent types shown from the same view as $B$. The afferent type numbers are shown in the top left corners. Scale bar (in $A$ ), $50 \mu \mathrm{m}$.

branching patterns of each pair when measured on a finer scale. First, as shown in Table 1, the total surface area of varicosities associated with each identified $\mathrm{M}$ afferent was one-half to onethird as large as the total varicosity surface area of the corresponding L afferent. Second, although $\mathrm{M}$ and $\mathrm{L}$ afferents of similar directional tuning projected to very similar neuropil locations, the arborizations did not overlap completely. In general, a greater proportion of each $\mathrm{M}$ afferent overlaped with each $\mathrm{L}$ afferent than vice versa. That is, the $\mathrm{M}$ afferent terminal arborizations were constrained to a subset of the total volume occupied by the corresponding afferent terminals. This is attributable primar- ily to the larger sizes of the $\mathrm{L}$ afferent arbors. Third, there were regions where $\mathrm{L}$ and $\mathrm{M}$ afferents were completely segregated from each other.

Figure 3 shows the combined projection patterns of all the $\mathrm{M}$ and $\mathrm{L}$ afferents. Each afferent arbor is represented by a cloud of points, color coded according to the peak directional tuning angle of the sensory neuron. Note that afferents with similar directional tuning overlap extensively and those with different directional tuning are spatially segregated. The arbors form a continuous representation of air current direction. The maps had a threedimensional, spiral-like shape in which directional tuning 
Figure 2. Left. Spatial relationships between pairs of $\mathrm{L}$ and $\mathrm{M}$ afferents. Shown is a computer reconstruction of the terminal arbors of three $\mathrm{M}$ afferents (blue) in the correct spatial relationships to $\mathrm{L}$ afferents of the same directional tuning (red). From top to bottom these are afferent types $10(A), 8(B)$, and $6(C)$. All afferents are shown from the dorsal view (the same view as in Fig. $1 B$ ) with the midline of the ganglion being the right edge of each panel. Note that the terminal fields of the $\mathrm{M}$ afferents are much smaller compared with the $\mathrm{L}$ afferents, and their terminals project to the same regions as the L afferents. There is some segregation between each pair of $\mathrm{L}$ and $\mathrm{M}$ afferents. The degree of such segregation varies among afferent types.

Figure 3. Right. Functional representation of direction in the terminal ganglion. $A$, Functional representation of direction formed by $\mathrm{L}$ afferents terminal arborizations. Each color cloud represents the average probability distribution of the terminal fields of afferents tuned to a particular wind direction. The directional tuning of each density cloud is represented by its color (shown also by the color wheel at the top right corner). The color wheel shows the direction with respect to the animal's body in the horizontal plane; yellow indicates air currents directed at the animal's head. $B$, Functional representation of direction by the $\mathrm{M}$ afferents (same view as in $A$ ). This figure demonstrates that the direction map of the $\mathrm{M}$ afferents is similar in structure to the $\mathrm{L}$ afferent map. The terminal fields of the $\mathrm{M}$ afferents overlap less with each other than the $\mathrm{L}$ afferents, making the map appear patchy and less tightly organized. $C$, Representation of direction by both $\mathrm{L}$ and $\mathrm{M}$ afferents within the terminal ganglion. $M$ and $\mathrm{L}$ afferents tuned to the same direction are spatially in register with one another, allowing direction as a whole to be represented continuously within the terminal ganglion.

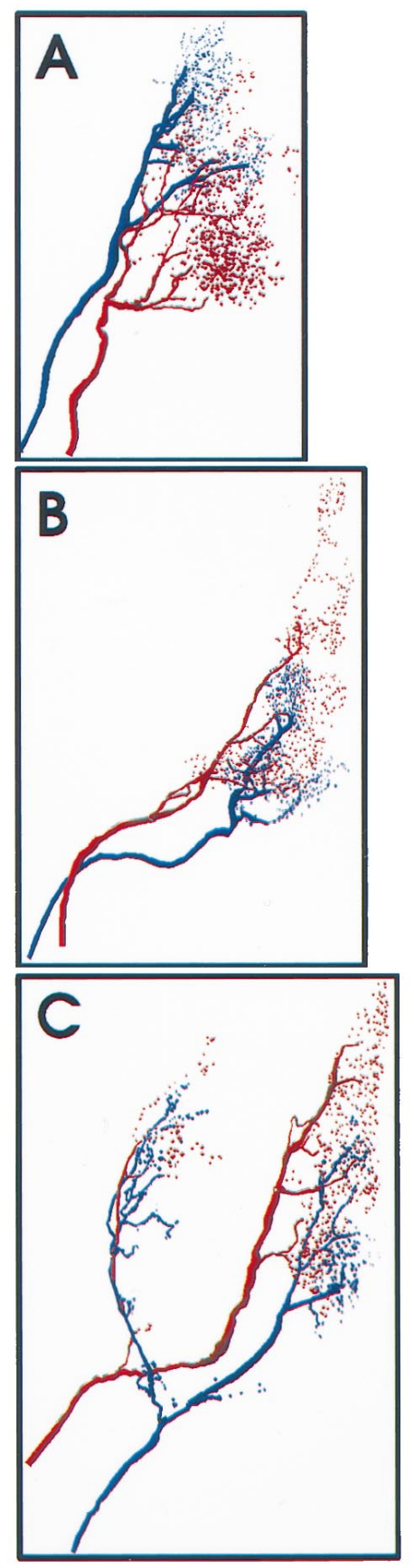

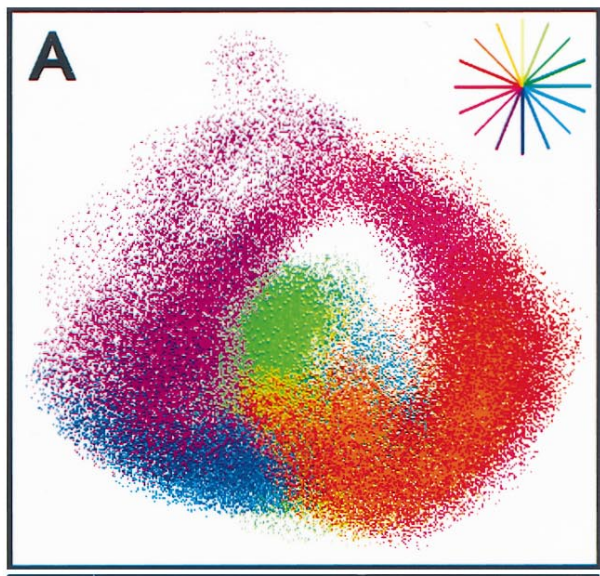
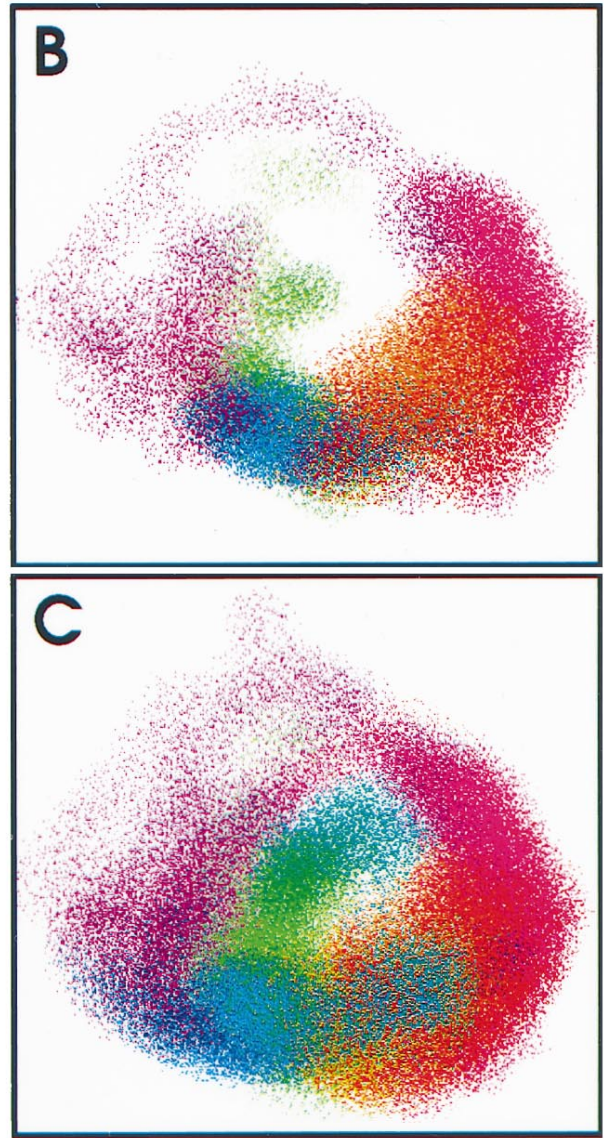

changed continuously around the spiral. The neural map of direction formed by the $\mathrm{M}$ afferents had the same continuous functional organization as the L afferent map (Fig. 3B). However, because of the smaller size of their arborizations, the $\mathrm{M}$ afferents formed a more patchy representation of air current direction. The projection patterns of $\mathrm{L}$ and $\mathrm{M}$ afferents are shown superimposed together in Figure $3 C$. Note that the directional maps formed by the long and medium-length hairs are aligned with one another and together form one continuous map of air current direction.

An important feature of the neural map of direction formed by the $\mathrm{L}$ afferents is the extensive anatomical overlap between afferent arbors from neurons with similar directional tuning (Jacobs and Theunissen, 1996). This relationship also held true in general for the $\mathrm{M}$ afferents, except that the degree of overlap was smaller. This is attributable to the smaller size of the M afferent arbors. In
Figure 4, the amounts of anatomical overlap between all paired combinations of $\mathrm{M}$ afferent arborizations were plotted as a function of the difference in their peak directional tuning and compared with the equivalent data from $\mathrm{L}$ afferents. Both plots show that there is approximately an inverse relationship between the amount of anatomical overlap and the separation in directional tuning for $\mathrm{L}$ and $\mathrm{M}$ afferents. There is significant anatomical overlap between many pairs of $\mathrm{M}$ afferents having peak directional tunings separated by $<90^{\circ}$. This relationship holds in general for both $\mathrm{L}$ and $\mathrm{M}$ afferents; however, the amount of overlap for $\mathrm{M}$ afferents is smaller than that for $\mathrm{L}$ afferents.

\section{Alignment of the $\mathbf{L}$ and $M$ afferent direction maps}

Previous studies have shown that the terminals of the $\mathrm{L}$ afferents are distributed nonuniformly throughout the map of air current 
Table 1. Anatomical characteristics of the identified sensory afferents

\begin{tabular}{|c|c|c|c|c|}
\hline Afferent type & $\begin{array}{l}\text { Area of the long hair } \\
\text { afferent }\left(\mu \mathrm{m}^{2}\right)\end{array}$ & $\begin{array}{l}\text { Area of the medium hair } \\
\text { afferent }\left(\mu \mathrm{m}^{2}\right)\end{array}$ & $\begin{array}{l}\text { Percent area of the } \\
\text { medium hair afferent } \\
\text { that overlaps with the } \\
\text { long hair afferent }\end{array}$ & $\begin{array}{l}\text { long hair afferent } \\
\text { that overlaps with the } \\
\text { medium hair afferent }\end{array}$ \\
\hline 1 & $1029.05 \pm 167.4$ & $714.14 \pm 16.31$ & $59.07 \pm 0.84$ & $40.95 \pm 0.57$ \\
\hline 2 & $1319.70 \pm 266.4$ & $1002.4 \pm 132.23$ & $51.36 \pm 0.70$ & $39.01 \pm 0.53$ \\
\hline 3 & $1113.04 \pm 277.8$ & $676.68 \pm 156.54$ & $45.00 \pm 0.89$ & $27.36 \pm 0.54$ \\
\hline 4 & $1392.86 \pm 147.9$ & $1052.13 \pm 204.45$ & $44.38 \pm 0.66$ & $33.52 \pm 0.50$ \\
\hline 5 & $1052.15 \pm 227.0$ & $847.55 \pm 102.03$ & $67.30 \pm 1.07$ & $54.21 \pm 0.86$ \\
\hline 6 & $1062.8 \pm 273.5$ & $829.72 \pm 210.10$ & $49.02 \pm 0.72$ & $38.27 \pm 0.56$ \\
\hline 7 & $1314.72 \pm 201.6$ & $643.78 \pm 71.71$ & $54.05 \pm 0.99$ & $26.74 \pm 0.49$ \\
\hline 8 & $1275.6 \pm 340.4$ & $747.22 \pm 158.63$ & $49.16 \pm 0.86$ & $28.80 \pm 0.50$ \\
\hline 9 & $1254.34 \pm 322.8$ & $955.76 \pm 91.88$ & $61.27 \pm 0.91$ & $46.68 \pm 0.69$ \\
\hline 10 & $1492.97 \pm 265.06$ & $648.61 \pm 85.65$ & $49.02 \pm 0.95$ & $21.30 \pm 0.41$ \\
\hline 11 & $1072.70 \pm 94.9$ & $708.69 \pm 149.64$ & $63.91 \pm 0.90$ & $42.22 \pm .060$ \\
\hline 13 & $1488.9 \pm 303.9$ & $835.35 \pm 92.05$ & $78.80 \pm 0.94$ & $43.65 \pm 0.72$ \\
\hline
\end{tabular}

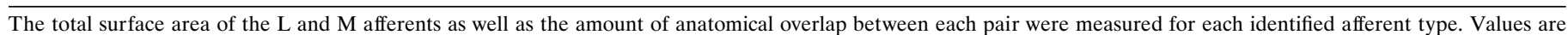

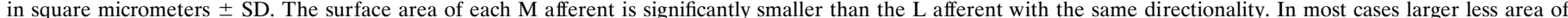

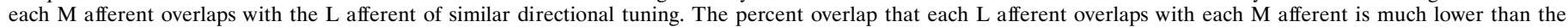
opposite case.

A

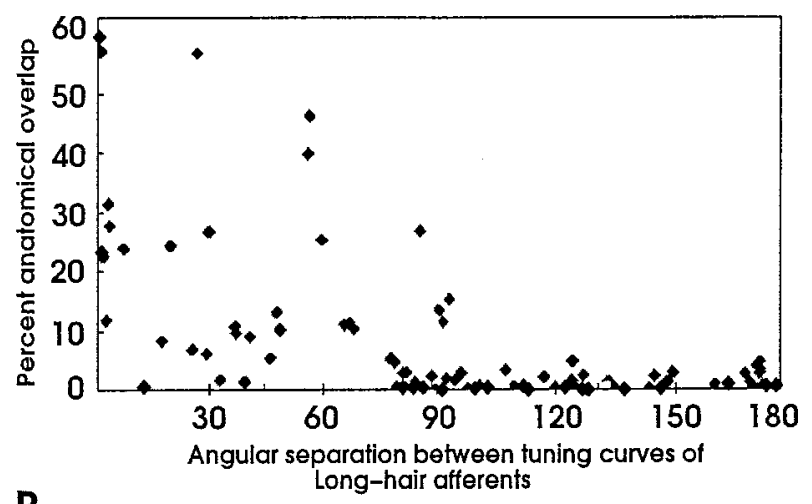

B

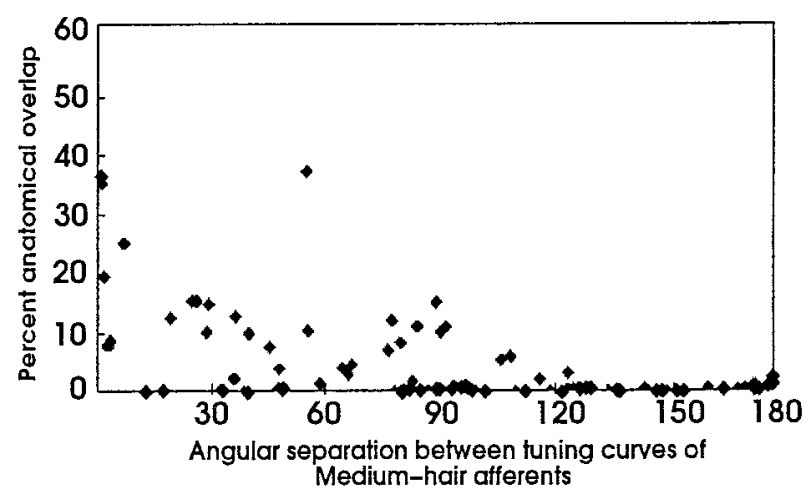

Figure 4. Correlation between anatomical overlap and differences in directional tuning. $A, B$, Relationship between the amount of anatomical overlap and angular separation among pairs of $\mathrm{L}$ and $\mathrm{M}$ afferents, respectively. Both graphs show that percent anatomical overlap between afferents decreases as the distance between their directional tuning increases. There is essentially no anatomical overlap between afferents whose directional tuning is $>90^{\circ}$. Note that percent anatomical overlap is less between pairs of $\mathrm{M}$ hair afferents compared with the same pairs of $\mathrm{L}$ afferents.

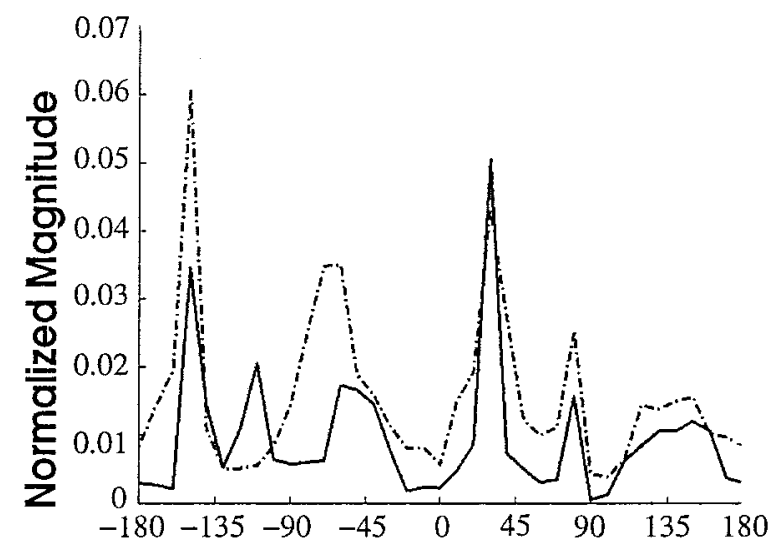

Peak Directional Tuning, degrees

rear left front right rear

Figure 5. Nonuniform representation of direction within different regions of the $\mathrm{L}$ and the $\mathrm{M}$ hair direction maps. Each point is the average magnitude of all directional vectors that are tuned to the same direction at that point in the map (shown on the $x$-axis). Directional tuning is represented with respect to body coordinates. There are five locations within the L direction map (dotted line) that have a greater magnitude compared with the rest of the map (data modified from Jacobs and Theunissen, 1996). Similar nonuniformity is seen for the M direction map (solid line). There are six locations in this map that have larger terminal density compared with the rest of the map. The peak density for the M afferents, in general, is much less than for the $\mathrm{L}$ afferents.

direction (Jacobs and Theunissen, 1996). The distribution of M afferent terminals, within the same neural space, was also nonuniform. Figure 5 shows the normalized magnitude of the afferent density distributions plotted as a function of net directional tuning angle within the map. The distribution of $\mathrm{L}$ afferent terminals (dotted line) was quite nonuniform, forming five large peaks of increased terminal density. The distribution of $\mathrm{M}$ afferents (solid line) follows this pattern very closely, with the exception of the presence of an additional peak between -90 and $-135^{\circ}$. This peak corresponds to an afferent (number 13) that was 
Figure 6. Anatomical segregation between two functional representations. $A$, Functional representation of stimulus direction by $\mathrm{L}$ and $\mathrm{M}$ afferents, color coded according to peak directional tuning with respect to body coordinates. Inset, The color wheel corresponds to the peak directional tuning in body coordinates of the subsets of afferents shown in $C-F$. $B$, Functional representation of stimulus frequency, represented by the $\mathrm{L}$ (red) and $\mathrm{M}$ (blue) afferent arborizations. $C-F$, Arborization patterns of subsets of $\mathrm{L}$ and $\mathrm{M}$ afferents tuned to different air current directions. $C$, Three $\mathrm{L}$ afferents and three $\mathrm{M}$ afferents with peak directional sensitivities near $-45^{\circ}$. $D$, Three $\mathrm{L}$ afferents and three $M$ afferents with peak directional sensitivities near $45^{\circ} . E$, Three L afferents and three $M$ afferents with peak directional sensitivities near $-135^{\circ} . F$, Three $\mathrm{L}$ afferents and three $M$ afferents with peak directional sensitivities near $135^{\circ}$.
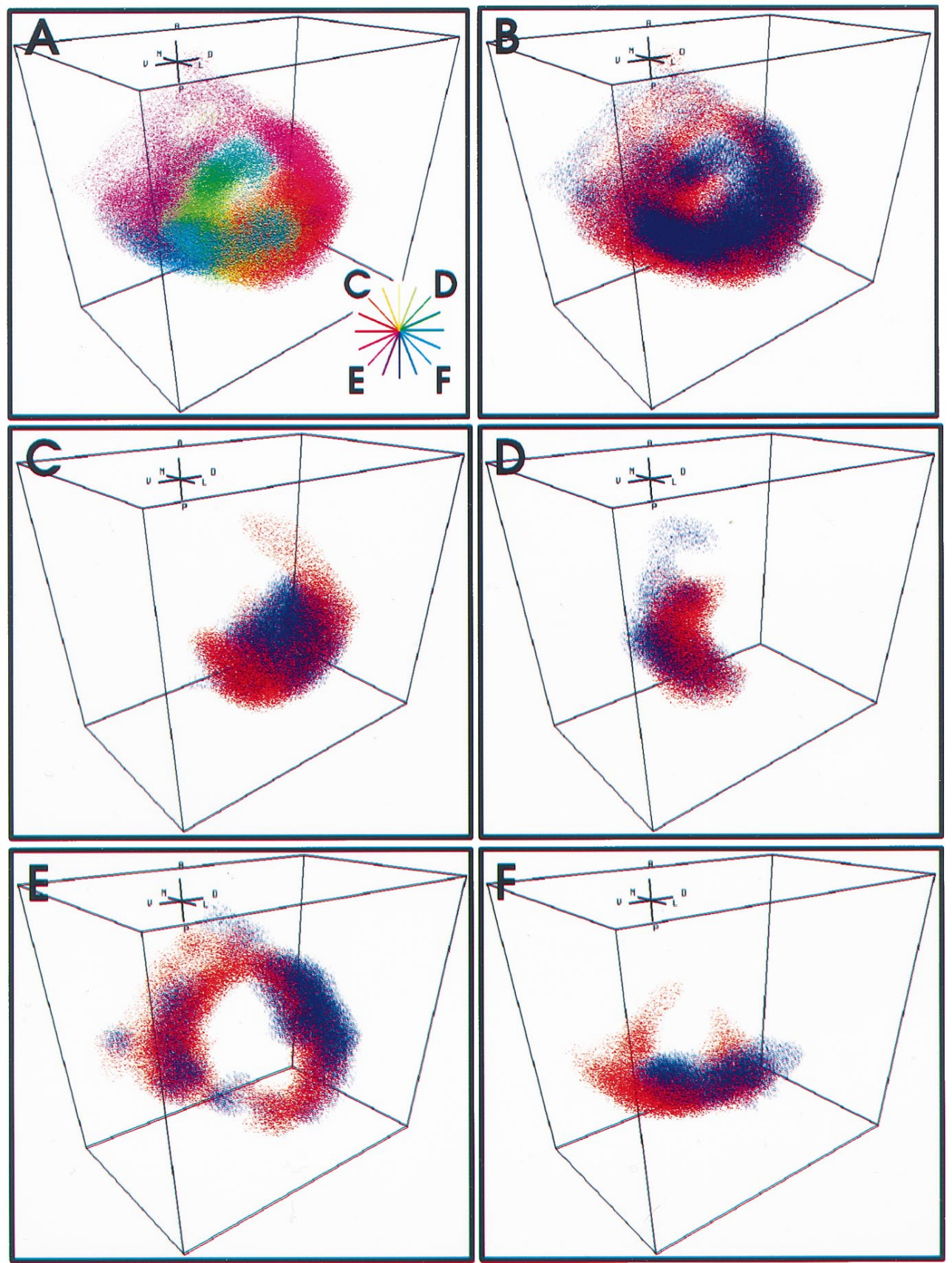

not included in the $\mathrm{L}$ afferent sample. Thus, both maps show a similar nonuniform distribution of terminal varicosities. In addition, Figure 5 demonstrates quantitatively the high degree of spatial alignment between the two maps of stimulus direction.

\section{Is a representation of air current frequency mapped anatomically?}

Figure 6 illustrates the degree of anatomical segregation between the $\mathrm{L}$ and $\mathrm{M}$ afferents with respect to their frequency sensitivity. The neural map of stimulus direction formed by all $\mathrm{L}$ and $\mathrm{M}$ afferents is shown in Figure $6 A$. In this panel, color is used to indicate peak directional sensitivity, as in the previous figures. The color wheel in the bottom right of this panel indicates the correspondence between the color in the map and the direction of peak sensitivity of the afferents projecting to that region. The remaining panels use a different color-coding scheme, so that spatial relationships between the $\mathrm{L}$ and $\mathrm{M}$ afferent populations can be seen more easily. Figure $6 B$ shows the projection pattern of all $\mathrm{L}$ afferents in red and all $\mathrm{M}$ afferents in blue. As indicated in the previous sections, there is a great deal of anatomical overlap between $\mathrm{L}$ and $\mathrm{M}$ afferents within the combined projection. However, there are small regions in which the $\mathrm{L}$ and $\mathrm{M}$ afferent populations are clearly segregated. By examining subsets of afferents that arborize in similar regions of the map, these regions of segregation can be seen more clearly. Figure $6 C$ shows the arborizations of three $\mathrm{L}$ afferents (red) and three $\mathrm{M}$ afferents (blue) that have peak directional sensitivities near $-45^{\circ}$ (as indicated in Figure $6 A$, small $C$ on the color wheel in the bottom right corner). The arborizations of three more subsets of afferents, with peak directional sensitivities clustered at $45,-135$, and $135^{\circ}$, are shown in Figure $6 D-F$, respectively. 

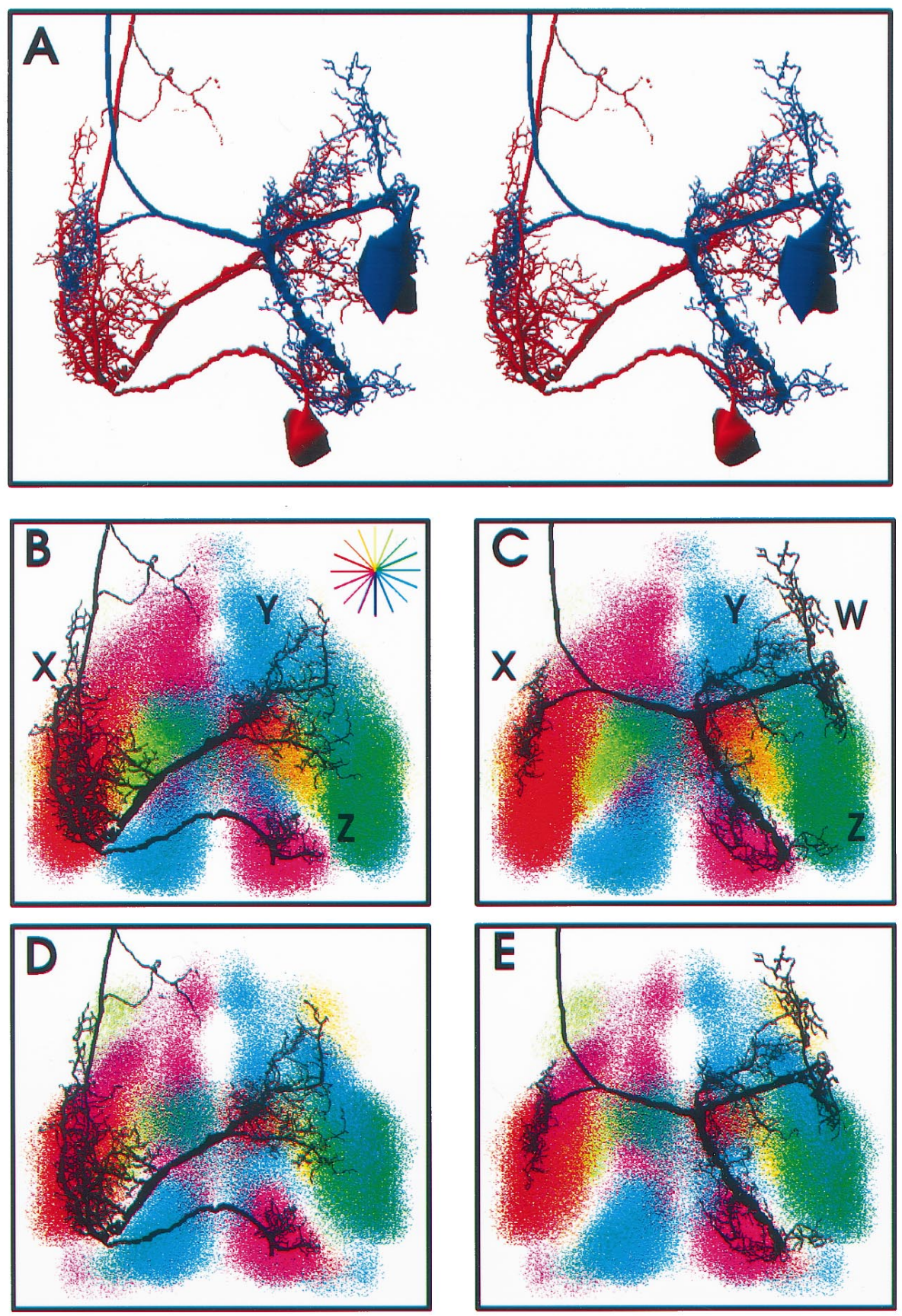

Figure 7. Distribution pattern of dendritic arbors of 10-3 and 9-3 interneurons with respect to the bilateral $\mathrm{M}$ and $\mathrm{L}$ hair direction maps. $A$, Stereo pair image of three-dimensional reconstructions of interneurons 10-3 (red) and 9-3 (blue) in the correct spatial relationships to each other. The dendritic arbors of these two interneurons overlap extensively and occupy similar regions within the cercal glomerulus. $B, D$, Anatomical relationships between 10-3 dendrites $\mathrm{X}, \mathrm{Y}$, and $\mathrm{Z}$ and different directional regions of the $\mathrm{M}$ and $\mathrm{L}$ direction maps, respectively. Each color cloud represents the probability density function of afferents tuned to a given direction. The directional sensitivity of each cloud is represented by its color shown along the direction wheel ( $B$, top right corner). The branching patterns of the interneurons are shown in black. The soma of each interneuron has been removed so as not to obscure the branching patterns. Note that the distribution pattern of 10-3 dendrites with respect to different directional regions are different between the two direction maps. For example, the portion of the $\mathrm{M}$ afferent direction map tuned to lateral wind directions ( green, top left corner of each panel) is spatially expanded over a larger area and falls outside dendrite $\mathrm{X}$ territory in the $\mathrm{M}$ afferent direction map. Note that there is more anatomical overlap between the same dendrite and the same directional region in the $\mathrm{L}$ afferent direction map. $C, E$, The anatomical relationships between dendritic arbors of 9-3 labeled W, X, Y, and $\mathrm{Z}$ and different directional regions of bilateral $\mathrm{L}$ hair $(C)$ and $\mathrm{M}$ hair $(E)$ direction maps. The amount and pattern of anatomical overlap between IN9-3 and the direction maps varies across directional regions. For example, the distribution pattern of dendrite $\mathrm{Y}$ in the region representing anterior wind direction (orange, top right corner) is different with respect to each of the two direction maps. Expansion of this region in the $\mathrm{M}$ afferent map allows more anatomical overlap with dendrite $\mathrm{Y}$ compared with that in the $\mathrm{L}$ afferent direction map.
Although there are regions of segregation between $\mathrm{M}$ and $\mathrm{L}$ afferents, there is no consistent pattern or axis of segregation indicating a continuous representation of stimulus frequency within the ensemble projection pattern. The continuous representation of direction is conserved in the combined projection pattern of $\mathrm{L}$ and $\mathrm{M}$ afferents, yet there does not appear to be a significant, systematic representation of stimulus frequency through any dimension.

\section{Are similar mechanisms used by primary sensory interneurons to extract directional and frequency tuning parameters from the neural map?}

The primary sensory neurons synapse with a group of $\sim 20$ uniquely identified sensory interneurons. Each of these interneurons has a unique morphology and a unique directional tuning, and each responds to a specific range of air current frequencies (Jacobs and Murphey, 1987; Miller et al., 1991; Theunissen et al., 1996). Previous studies suggest that the directional tuning characteristics of each interneuron are determined by its anatomical structure: the position of each neuron's dendritic branches within the afferent map of air current direction determines the set of excitatory inputs it can receive and thus shapes its directional tuning characteristics (Bacon and Murphey, 1984; Jacobs and Murphey, 1987; Troyer et al., 1994). Could a similar anatomybased mechanism determine the differential frequency sensitivities of the interneurons? That is, could the anatomy of the dendritic arbors of the different interneurons allow differential connectivity with afferents tuned to different frequency sensitivities? 
10-3X with L/M afferents

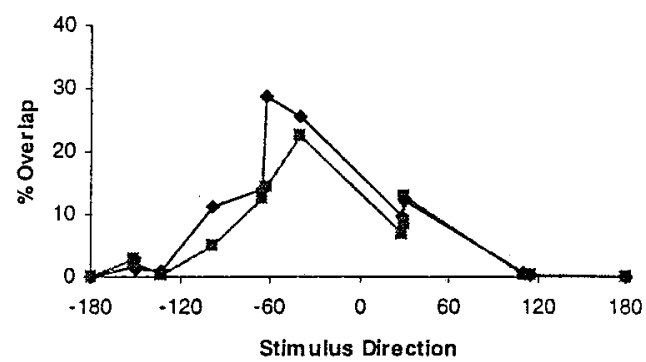

10-3Y with $L / M$ afferents

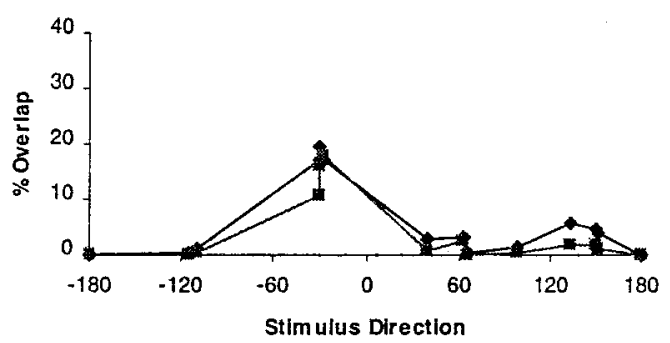

10-3Z with L/M afferents

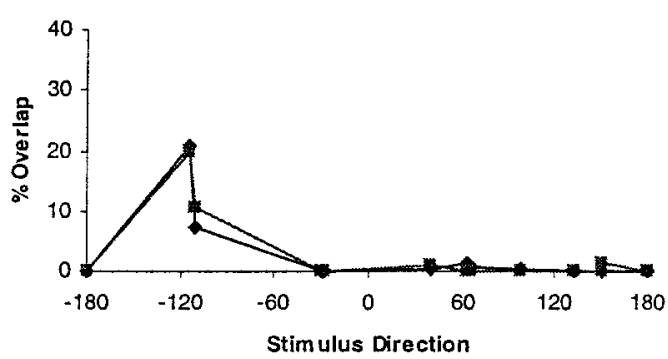

9-3X with LM Afferents

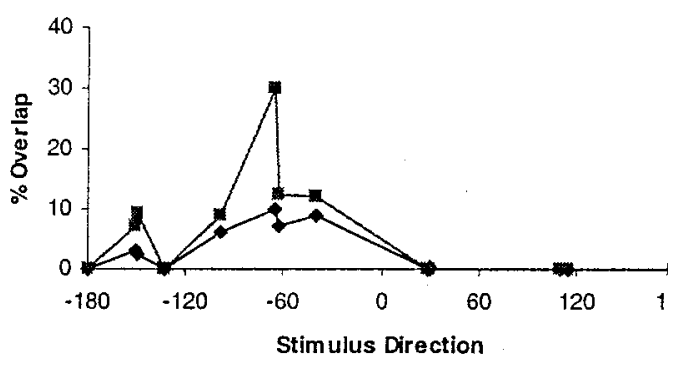

9-3y with L/M Afferents

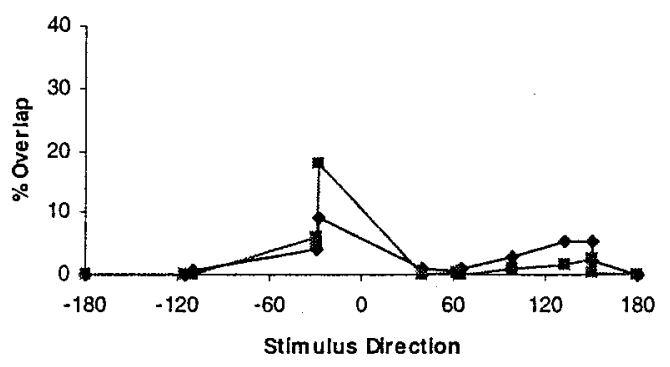

9-3Z with L/M Afferents

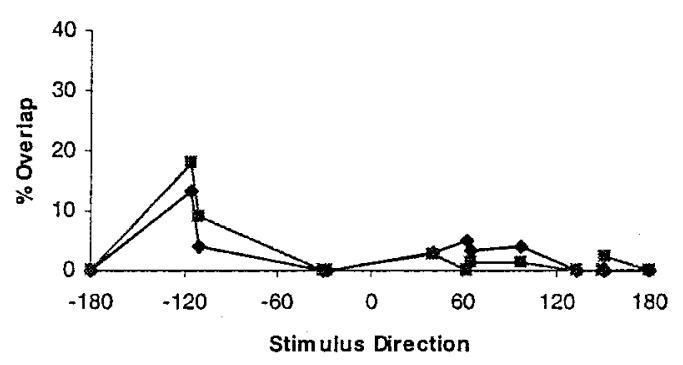

9-3W with L/M Afferents

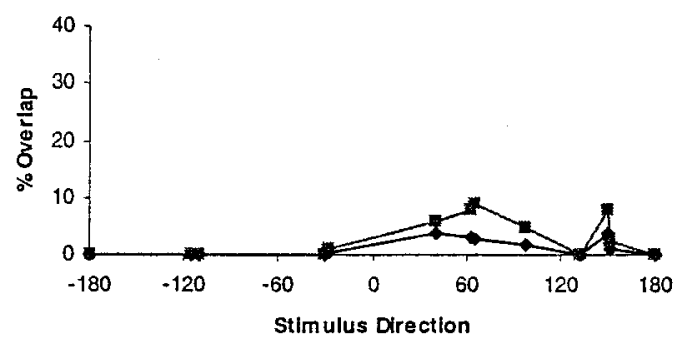

Figure 8. Quantitative analysis of anatomical relationships between INs 10-3 and 9-3 and directional regions of the $\mathrm{L}$ and $\mathrm{M}$ afferent direction maps (triangles, L afferents; squares, $\mathrm{M}$ afferents). Left column, Percent anatomical overlap among X, Y, and Z dendrites of the 10-3 interneuron and different regions of the direction maps. The peak directional sensitivity of each dendrite corresponds closely with the amount of anatomical overlap it has with the afferents (whether $\mathrm{L}$ or $\mathrm{M}$ ) that have the same directional sensitivities. Note that dendrites of the 10-3 overlap slightly more with $\mathrm{L}$ afferents than with the M afferents with the same directional sensitivity. Right column, Percent anatomical overlap among each dendrite of the 9-3 interneuron (X, Y, $\mathrm{W}, \mathrm{Z}$ ) and different regions of the direction maps. The peak directional sensitivity of each dendrite correlates closely with the amount of its anatomical overlap with afferents that have the same peak directional sensitivity.

To answer this question, the amount of anatomical overlap between $\mathrm{L}$ and $\mathrm{M}$ afferents with two different identified interneurons was measured. These two interneurons are known to have similar directional tuning properties but are sensitive to different ranges of air current frequency. Interneuron 10-3 (Fig. $7 A$, red) has a frequency sensitivity range similar to the $\mathrm{L}$ afferents, and interneuron 9-3 (Fig. 7A, blue) has a frequency sensitivity range similar to that of the M afferents (Miller et al., 1991; Theunissen et al., 1996). Other studies have suggested that these differences in frequency tuning might arise from differential connectivity between $\mathrm{M}$ and $\mathrm{L}$ afferents (Chiba et al., 1992).

The dendritic arborizations of these two interneurons overlap extensively, occupying similar areas of neuropil (Fig. $7 A$, stereo pair). The pattern of anatomical overlap between interneurons 
10-3 and 9-3 and the M and L direction maps is shown in Figure $7 B-E$. Although both interneurons appear to sample very similar areas of the two maps, there are subtle differences between the two arborization patterns. For example, dendrite X of 10-3 overlaps almost equally with areas representing lateral and anterior directions (green and red, respectively) in the L direction map. However, that same dendrite overlaps less with these two regions in the $\mathrm{M}$ direction map. Conversely, dendrite $\mathrm{Y}$ of interneuron 9-3 overlaps more with areas representing lateral wind directions in the $\mathrm{M}$ direction map than in the $\mathrm{L}$ map.

To quantify these anatomical observations, the amount of anatomical overlap between each of the interneuron dendrites and each of the afferents was measured (Fig. 8). The results of these calculations demonstrate two points. First, corresponding dendrites of each interneuron do, indeed, overlap with the same sets of afferents. Second, the anatomical overlap between the interneurons and both populations of afferents is statistically indistinguishable. Thus, the degree of differential connectivity emerging from the physical segregation illustrated in Figure 7 is not significant, and this small degree of segregation could not be responsible for the observed frequency selectivity of the interneurons.

\section{DISCUSSION}

\section{Representation of multiple sensory parameters}

Representations of multiple sensory stimulus parameters have been demonstrated in a variety of systems (Middlebrooks et al., 1980; Middlebrooks and Pettigrew, 1981; Shipp and Zeki, 1985; Hubel and Livingstone, 1987; Clarey et al., 1994). This paper demonstrates how two different sensory stimulus parameters, direction and frequency, are organized anatomically in the cricket cercal sensory system. Afferents tuned to similar air current directions segregate from one another to form a continuous representation of stimulus direction within the terminal ganglion (Jacobs and Theunissen, 1996). However, the extensive overlap between $\mathrm{L}$ and $\mathrm{M}$ afferents indicates that there is no obvious segregation of their arbors according to frequency sensitivity. Thus, stimulus frequency is not mapped in any recognizable coordinate system. Rather, information about stimulus frequency is distributed throughout the neural map of stimulus direction.

This anatomical organization has several functional consequences. Given that direction and frequency parameters are encoded by the same sets of afferents, the same regions of neuropil will be activated by air currents from a given direction regardless of the stimulus frequency. Thus the direction of the stimulus is preserved throughout the entire frequency range of stimuli. In contrast, for a given frequency, the area of neuropil activated by a sensory stimulus will change systematically as a function of stimulus direction. Most biologically relevant stimuli are composed of a range of stimulus frequencies; however, fewer may be composed of stimuli in which direction is changing dynamically.

\section{Comparison with other sensory systems}

The anatomical organization of the cricket system is quite different from some neural maps found in the auditory system. In owls, cats, and bats different stimulus parameters are mapped in orthogonal coordinate systems (Knudsen and Konishi, 1978; O'Neill and Suga, 1982; Middlebrooks and Knudsen, 1984; for review, see Knudsen et al., 1987; Carr, 1993). For example, in the primary auditory cortex, stimulus intensity is mapped orthogonal to stimulus frequency (Middlebrooks et al., 1980). This functional organization results in a unique combination of stimulus parameters mapped at any one neuropil location. In the nucleus magnocellularis of the barn owl, interaural phase difference is mapped along one axis, and stimulus frequency is mapped along another axis (Carr and Konishi, 1990). This arrangement is functionally similar to the cricket system, in that a map of interaural time differences exists for each range of stimulus frequencies.

The functional organization of the cercal cricket system is perhaps more similar to V1 in the cat visual cortex. Several researchers have demonstrated the existence of multiple functional maps in the cat visual cortex (Hubener et al., 1997; Shoham et al., 1997). They and others have demonstrated a continuous functional map of orientation preference (Arieli et al., 1995; Bosking et al., 1997) and, more recently, maps of spatial frequency. The maps of spatial frequency are not continuous representations but are patchy and divided into two domains, one tuned to low spatial frequencies and one to high spatial frequencies. We predict a very similar functional organization in the cricket system. The afferent projection scheme in the cricket cercal system results in a continuous map of stimulus direction that has structural attributes very similar to those of the pinwheels seen in the visual cortical map (Bonhoeffer and Grinvald, 1993). In contrast, the representation of stimulus frequency in the cricket cercal map does not show any clear anatomical segregation. The cercal map of stimulus frequency is very likely to be patchy and discontinuous. Low-frequency air current stimuli should elicit large patches of activity, whereas high stimulus frequencies should elicit relatively small patches of activity. This prediction is based on the fact that afferents sensitive to low air current velocities have large overlapping arborizations, and afferents sensitive to high stimulus frequencies have much smaller nonoverlapping arborizations. Thus, in both the visual system and the cercal system, one functional parameter is mapped continuously (visual stimulus orientation/air current direction) and another functional parameter is not (visual spatial frequency/air current frequency).

\section{Extraction of information from map by interneurons: two different mechanisms}

There is good evidence, based on physiological studies, that interneuron 10-3 receives a majority of its afferent input from the longest hairs on the cercus (Shimozawa and Kanou, 1984a,b; Chiba et al., 1992; Theunissen et al., 1996). Interneuron 10-3 is tonically active under ambient conditions (as are the $\mathrm{L}$ afferents) and tuned to a frequency range very similar to that of the $\mathrm{L}$ afferents. Conversely, interneuron 9-3 is insensitive to the lowest frequency ranges (as are $\mathrm{M}$ afferents) and is silent under ambient conditions, suggesting that the bulk of its afferent inputs may come from M afferents (Miller et al., 1991).

However, our results indicate that anatomical relationships between afferents and interneurons do not play a significant role in determining the frequency-tuning characteristics of the interneurons. Two interneurons, interneuron 10-3 (tuned to low frequencies) and interneuron 9-3 (tuned to higher frequencies), have nearly equal anatomical access to $\mathrm{L}$ and $\mathrm{M}$ afferents. Thus, if frequency tuning of interneurons depends on selective connections with afferents tuned to a specific dynamic range of stimuli, then mechanisms other than anatomical segregation must play a role.

Possible mechanisms that could account for these differences in frequency tuning fall into two general categories. First, selective 
connectivity between interneurons and restricted subsets of afferents having specific frequency-tuning characteristics (e.g., between 10-3 and L afferents) could be achieved through mechanisms other than differential overlap with segregated inputs. Presumably, mechanisms for the recognition of molecular labels or for activity-dependent strengthening of certain synapses would need to exist to allow such specificity of connection. As a second general alternative, connectivity between interneurons and afferents could be nonselective, with the frequency characteristics of the interneurons established through some kind of "frequency filtering" of those inputs within the interneuron. These two possibilities should be considered as exclusive of one another.

Considerable evidence supports this latter possibility: afferents with different frequency-tuning properties have been demonstrated to make connections onto the same interneuron (Shepherd and Murphey, 1986; Chiba et al., 1988; Davis and Murphey 1993, 1994). Davis and Murphey (1993, 1994) suggested that a single afferent can connect to two different interneurons yet make synapses with very different properties: either habituating or facilitating. These authors suggest that "synaptic matching," although presynaptic in origin, may involve retrograde feedback from the postsynaptic cell. Thus the postsynaptic cell may determine the presynaptic character of its inputs and thereby sculpt its frequency sensitivity to air currents.

The frequency-tuning characteristics of interneurons have been shown to display obvious bandpass characteristics (Theunissen et al., 1996). The tuning curve of interneuron 10-3 is much narrower than the tuning curves of the L afferents thought to form the bulk of their synaptic input. Thus, at least some of the shaping of the frequency-encoding properties of the cells cannot be caused by selective connectivity and must be caused by intrinsic filtering. Possible mechanisms for this intrinsic filtering include (1) lowpass attenuation of synaptic currents between their synaptic input sites and the spike-initiating zone, attributable to the passive electrotonic properties of the dendrites; and (2) active amplification of higher-frequency components by voltage-dependent dendritic conductances (Horner et al., 1997). These biophysical mechanisms play an important role in determining the frequency sensitivity of interneurons in the fly visual system. (Borst and Eglehaaf, 1992, 1994). These properties have been shown to depend on the particular mix of voltage-gated ion channels in the dendrites of the different cell types (Haag and Borst, 1996; Haag et al., 1997).

Our results present an interesting challenge for understanding the cellular mechanisms underlying extraction of different stimulus features from this neural map by interneurons in the cercal system. Clearly, the extraction of information about stimulus frequency from this map must involve a combination of selective connectivity mechanisms, retrograde regulation of synaptic release properties, and/or differences in biophysical properties of the interneurons.

\section{REFERENCES}

Arieli A, Shoham D, Hildesheim R, Grinvald A (1995) Coherent spatiotemporal patterns of ongoing activity revealed by real-time optical imaging coupled with single-unit recording in the cat visual cortex. J Neurophysiol. 73:2072-2093.

Bacon JP, Altman JS (1977) A silver intensification method for cobalt filled neurons in whole mount preparations. Brain Res 138:359-363.

Bacon JP, Murphey RK (1984) Receptive fields of cricket giant interneurons are related to their dendritic structures. J Physiol (Lond) 352:601-623.

Bonhoeffer T, Grinvald A (1993) The layout of iso-orientation domains in area 18 of cat visual cortex: optical imaging reveals a pinwheel-like organization. J Neurosci 13:4157-4180.

Borst A, Egelhaaf M (1992) In vivo imaging of calcium accumulation in fly interneurons as elicited by visual motion stimulation Proc Natl Acad Sci USA 89:4139-4143.

Borst A, Egelhaaf M (1994) Dendritic processing of synaptic information by sensory interneurons Trends Neurosci 12:257-263.

Bosking WH, Zhang Y, Schofield B, Fitzpatrick D (1997) Orientation selectivity and the arrangement of horizontal connections in tree shrew striate cortex. J Neurosci 17:2112-2127.

Carr CE (1993) Processing of temporal information in the brain. Annu Rev Neurosci 16:223-243.

Carr CE, Konishi M (1990) A circuit of detection of interaural time differences in the brainstem of the barn owl. J Neurosci 10:3227-3246.

Chiba A, Shepherd D, Murphey RK (1988) Synaptic rearrangement during postembryonic development in the cricket. Science 240:901-905.

Chiba A, Kamper G, Murphey RK (1992) Response properties of interneurons of the cricket cercal system are conserved in spite of changes in peripheral receptors during maturation. J Exp Biol 164:1-22.

Clarey JC, Borner P, Imig TJ (1994) Functional organization of sound direction and sound pressure level in primary auditory cortex of the cat. J Neurophysiol 72:2383-2405.

Davis GW, Murphey RK (1993) A role for postsynaptic neurons in determining presynaptic release properties in the cricket CNS: evidence for retrograde grade control of facilitation. $J$ Neurosci 13:3827-3838.

Davis GW, Murphey RK (1994) Retrograde signaling and the development of transmitter release properties in the invertebrate nervous system. J Neurobiol 25:740-756.

Egelhaaf M, Borst A (1995) Calcium accumulation in visual interneurons of the fly: stimulus dependence and relationship to membrane potential. J Neurophysiol 73:2540-2552.

Haag J, Borst A (1996) Amplification of high-frequency synaptic inputs by active dendritic membrane processes. Nature 379:639-641.

Haag J, Theunissen F, Borst A (1997) The intrinsic electrophysiolgical characteristics of fly lobula plate tangential cells. II. Active membrane properties. J Comput Neurosci 4:349-369.

Horner M, Kloppenburg P, Heblich R (1997) Characterization of ionic currents from identified cricket giant interneurons in primary cell culture. In: Proceedings of the 25th Gottingen Neurobiology Conference, Vol 2, p 780 .

Hubel DH, Livingstone MS (1987) Segregation of form, color, and streopsis in primate area 18. J Neurosci 7:3378-3415.

Hubener M, Shoham D, Grinvald A, Bonhoeffer T (1997) Spatial relationships among three columnar systems in cat area 17 . J Neurosci 17:9270-9284.

Jacobs GA, Murphey RK (1987) Segmental origins of the cricket giant interneuron system. J Comp Neurol 265:145-157.

Jacobs GA, Nevin R (1991) Anatomical relationships between sensory afferent arborizations in the cricket cercal system. Anat Rec 231:563-572.

Jacobs GA, Theunissen FE (1996) Functional organization of a neural map in the cricket cercal sensory system. J Neurosci 16:769-784.

Jacobs GA, Miller JP, Murphey RK (1986) Cellular mechanisms underlying directional sensitivity of an identified sensory interneuron. J Neurosci 6:2298-2311.

Johnson SE, Murphey RK (1985) The afferent projection of mesothoracic bristle hairs in the cricket Acheta domesticus. J Comp Physiol [A] 156:369-379.

Kamper G, Kleindienst HU (1990) Oscillation of cricket sensory hairs in a low-frequency sound field. J Comp Physiol [A] 167:193-200.

Kanou M, Shimozawa TA (1984) Threshold analysis of cricket cercal interneurons by an alternating air-current stimulus. J Comp Physiol [A] 154:357-365.

Knudsen EI, Konishi M (1978) A neural map of auditory space in the owl. Science 200:795-797.

Knudsen EI, Sascha DL, Esterly SD (1987) Computational maps in the brain. Annu Rev Neurosci 10:41-65.

Landolfa MA, Jacobs GA (1995) Direction sensitivity of the filiform hair population of the cricket cercal system. J Comp Physiol [A] 177:759-766.

Landolfa MA, Miller JP (1995) Stimulus-response properties of cricket cercal filiform receptors. J Comp Physiol [A] 177:747-757.

Middlebrooks JC, Knudsen EI (1984) A neural code for auditory space in the cat's superior colliculus. J Neurosci 4:2621-2634. 
Middlebrooks JC, Pettigrew JD (1981) Functional classes of neurons in primary auditory cortex of the cat distinguished by sensitivity to sound location. J Neurosci 1:107-120.

Middlebrooks JC, Dykes RW, Merzenich MM (1980) Binaural response-specific bands in primary auditory cortex (AI) of the cat: topographical organization orthogonal to isofrequency contours. Brain Res 181:31-48.

Miller JP, Jacobs GA, Theunissen FE (1991) Representation of sensory information in the cricket cercal sensory system. I. Response properties of the primary interneurons. J Neurophysiol 66:1680-1689.

O'Neill WE, Suga N (1982) Encoding of target-range information and its representation in the auditory cortex of the mustached bat. J Neurosci 2:17-24.

Roddey JC, Jacobs GA (1996) Information theoretic analysis of dynamical encoding by filiform mechanoreceptors in the cricket cercal system. J Neurophysiol 75:1365-1376.

Shepherd D, Murphey RK (1986) Competition regulates the efficacy of an identified synapse in crickets. J Neurosci 6:3152-3160.

Shimozawa T, Kanou M (1984a) Varieties of filiform hairs: range fractionation by sensory afferents and cercal interneurons of the cricket. J Comp Physiol [A] 155:485-493.

Shimozawa T, Kanou M (1984b) The aerodynamics and sensory physi- ology of range fractionation in the cercal filiform sensilla of the cricket Gryllus bimaculatus. J Comp Physiol [A] 155:495-505.

Shipp S, Zeki S (1985) Segregation of pathways leading from area V2 to areas V4 and V5 of macaque monkey visual cortex. Nature 315:322-325.

Shoham D, Hubener M, Schulze S, Grinvald A, Bonhoeffer T (1997) Spatio-temporal frequency domains and their relation to cytochrome oxidase staining in cat visual cortex. Nature 385:529-533.

Theunissen FE, Miller MP (1991) Representation of sensory information in the cricket cercal sensory system. II. Information theoretic calculation of system accuracy and optimal tuning-curve widths of four primary interneurons. J Neurophysiol 66:1690-1703.

Theunissen FE, Roddey JC, Stufflebeam S, Clague H, Miller JP (1996) Information theoretic analysis of dynamical encoding by four primary sensory interneurons in the cricket cercal system. J Neurophysiol 75:1345-1376.

Troyer TW, Levin JE, Jacobs GA (1994) Construction and analysis of a data base representing a neural map. Microsc Res Tech 29:329-343.

Warland D, Landolfa MA, Miller JP, Bialek W (1991) Reading between the spikes in the cercal filiform hair receptors of the cricket. In: Analysis and modeling of neural systems (Eeckman FH, ed), pp 327333. Boston: Kluwer. 